\title{
BMJ Open Disease and demography: a systems- dynamic cohort-component population model to assess the implications of disease-specific mortality targets
}

\author{
Muhammad Jami Husain (D) , Biplab Kumar Datta, Deliana Kostova
}

To cite: Husain MJ, Datta BK, Kostova D. Disease and demography: a systemsdynamic cohort-component population model to assess the implications of disease-specific mortality targets. BMJ Open 2021;11:e043313. doi:10.1136/ bmjopen-2020-043313

- Prepublication history and additional supplemental material for this paper are available online. To view these files, please visit the journal online (http://dx.doi.org/10.1136/ bmjopen-2020-043313).

Received 03 August 2020 Revised 01 March 2021 Accepted 25 April 2021
Check for updates

(c) Author(s) (or their employer(s)) 2021. Re-use permitted under CC BY-NC. No commercial re-use. See rights and permissions. Published by BMJ.

Division of Global Health Protection, Centers for Disease Control and Prevention, Atlanta, Georgia, USA

Correspondence to Dr Muhammad Jami Husain; MHusain@cdc.gov

\section{ABSTRACT}

Introduction The 2015 Sustainable Development Goals include the objective of reducing premature mortality from major non-communicable diseases (NCDs) by one-third by 2030. Accomplishing this objective has demographic implications with relevance for countries' health systems and costs. However, evidence on the system-wide implications of NCD targets is limited.

Methods We developed a cohort-component model to estimate demographic change based on user-defined disease-specific mortality trajectories. The model accounts for ageing over 101 annual age cohorts, disaggregated by sex and projects changes in the size and structure of the population. We applied this model to the context of Bangladesh, using the model to simulate demographic outlooks for Bangladesh for 2015-2030 using three mortality scenarios. The 'status quo' scenario entails that the disease-specific mortality profile observed in 2015 applies throughout 2015-2030. The 'trend' scenario adopts age-specific, sex-specific and disease-specific mortality rate trajectories projected by WHO for the region. The 'target' scenario entails a one-third reduction in the mortality rates of cardiovascular disease, cancer, diabetes and chronic respiratory diseases between age 30 and 70 by 2030 .

Results The status quo, trend and target scenarios projected 178.9, 179.7 and 180.2 million population in 2030, respectively. The cumulative number of deaths during 2015-2030 was estimated at 17.4, 16.2 and 15.6 million for each scenario, respectively. During 2015-2030, the target scenario would avert a cumulative 1.73 million and 584000 all-cause deaths compared with the status quo and trend scenarios, respectively. Male life expectancy was estimated to increase from 71.10 to 73.47 years in the trend scenario and to 74.38 years in the target scenario; female life expectancy was estimated to increase from 73.68 to 75.34 years and 76.39 years in the trend and target scenarios, respectively.

Conclusion The model describes the demographic implications of NCD prevention and control targets, estimating the potential increase in life expectancy associated with achieving key NCD reduction targets. The results can be used to inform future health system needs and to support planning for increased healthcare coverage in countries.

\section{Strengths and limitations of this study}

- The model provides an understanding of how changes in disease-specific mortality may contribute to the demographic outlook of countries by simulating demographic evolution paths corresponding to prespecified mortality rate outlooks.

- The model tracks population outcomes at a highly disaggregated level and can produce consistent and comparable cross-country estimates for a set of demographic indicators.

- The model uses established principles about the dynamics of the population process and can be flexibly adapted to the intended disaggregation schemes of population cohorts and disease categories.

- The cohort-component method does not explicitly incorporate socio-economic determinants of population change.

- The model outcomes are based on conditional calculations producing outlooks for a set of demographic indicators under a particular set of reasonable assumptions.

\section{INTRODUCTION}

Changes in population size and demographic composition have broad economic and social implications. Informed decisions regarding population-level policies and interventions hinge on robust population projections that delineate the dynamic interplay of demographic processes such as fertility, mortality and migration. Generating counts for population cohorts of interest determines investment in sectors like health, education, infrastructure and others. ${ }^{12}$

We present a cohort-component population projection model to assess demographic changes associated with changes in the distribution of causes of death. Current population projections reflect a variety of assumptions about fertility, mortality and migration..$^{3-6}$ For instance, the UN produces eight variants of population projections, five of which are based on different trajectories of fertility, 


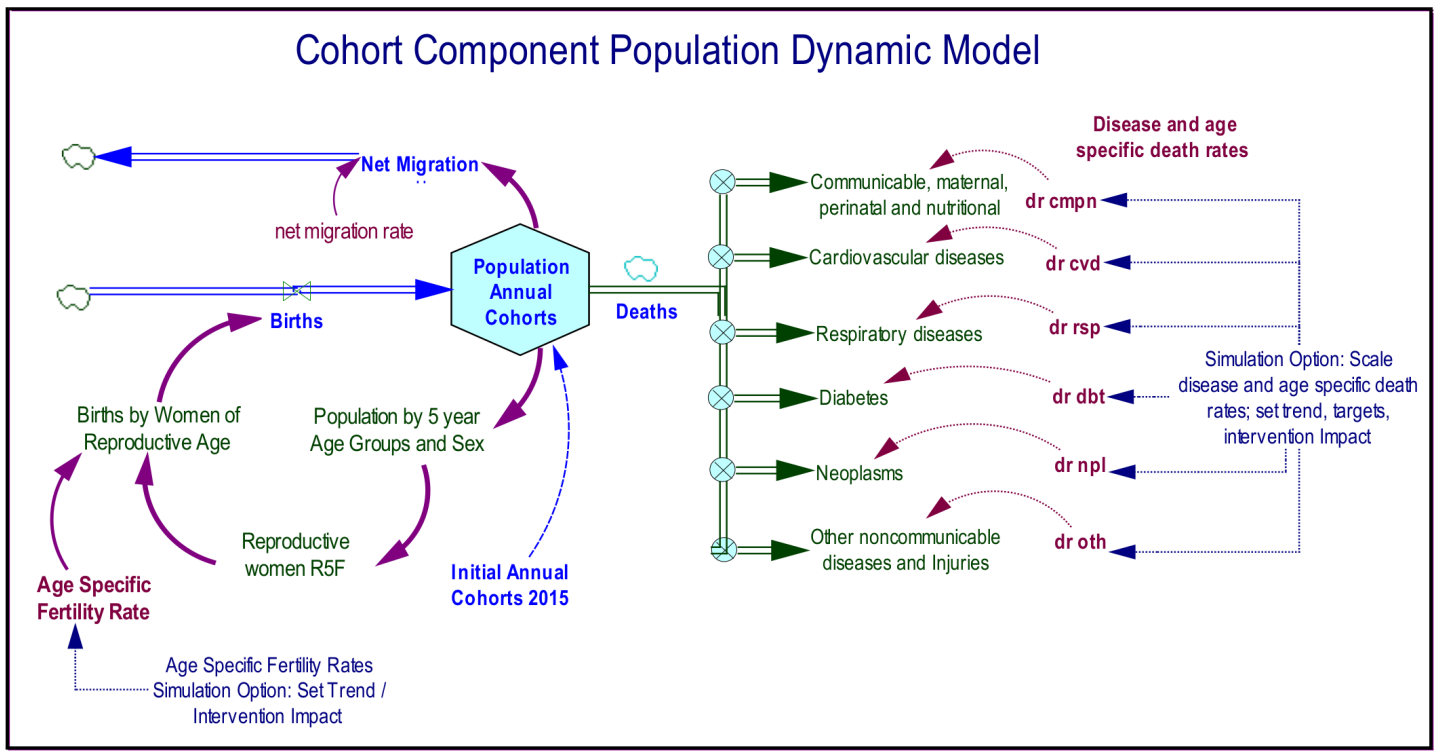

Figure 1 Overview of the cohort-component population model: stock, flows and simulation options. Note: The model is developed using the Vensim DSS (V.8) simulation platform. cmpn, communicable, maternal, perinatal and nutritional conditions; cvd, cardiovascular diseases; dbt, diabetes mellitus; dr, death rate; npl, neoplasms; oth, other non-communicable diseases and injuries; rsp, respiratory disease.

while mortality assumptions are determined by probabilistic trends of life expectancy at birth, and international migration is assumed either constant or zero. ${ }^{3}$ The existing population projection models typically emphasise the role of fertility but do not provide an understanding of how changes in disease-specific mortality rates may contribute to the demographic outlook of countries.

Preventable deaths and disability caused by communicable diseases, maternal, perinatal and nutritional conditions (CMPN), non-communicable diseases (NCDs) and injuries constitute core concerns across nations. Among these, cardiovascular diseases (CVDs) are in the lead, accounting for 15.2 million deaths of all 56.9 million deaths worldwide in $2016 .{ }^{7}$ Given the rising significance of NCDs in global health, the 2030 Sustainable Development Goals (SDGs) aim to reduce premature mortality from the four major NCDs (CVDs, diabetes, cancer and respiratory diseases) by one-third by 2030, relative to 2015 level. ${ }^{8}$ With the adoption of the WHO Global NCD Action Plan by the World Health Assembly in 2013, the WHO Member States agreed on a time-bound voluntary target of attaining a $25 \%$ relative reduction in overall mortality from the four leading NCDs by $2025 .{ }^{9}$ In a similar vein, the WHO 2013 Global Program of Work (GPW 2013) set the target of $20 \%$ relative reduction in the premature mortality (age 30-70 years) from these NCDs between 2019 and $2023 .^{10}$ These objectives occur in the context of many budgetary and planning constraints that affect low-income and middle-income countries (LMICs). Further, variations in the incidence and prevalence of diseases across sex and age cohorts require policymakers to formulate targeted interventions and policies. Understanding the evolution of different age-cohorts resulting from shifts in disease-specific mortality over time can inform the resource needs for national scale-up of interventions to attain SDG health targets.

The dynamic population projection model in this study simulates a range of demographic evolution paths corresponding to pre-specified disease-specific mortality outlooks. The results provide demographic information needed to plan for services to meet future demands of different segments of the population. Although the model in this study is applied to Bangladesh, it is replicable across different countries and can serve as a tool for planners to simulate user-defined scenarios corresponding to assumed fertility, mortality, and international migration trajectories.

Over the last several decades, Bangladesh has made substantial progress in disease prevention and control of childhood communicable diseases, but NCDs have emerged as the primary cause of death and disability in the country. ${ }^{112}$ In response, the Government of Bangladesh has formulated an NCD action plan to reduce NCDs and associated risk factors through a multisectoral coordinated approach. ${ }^{13}$ Bangladesh NCD prevention and control targets are consistent with the 2030 SDGs and with the WHO South-East Asia regional NCD 2025 objectives of reducing by $25 \%$ premature mortality from CVDs, diabetes, respiratory diseases and cancer. ${ }^{13}{ }^{14}$ Attainment of these targets entails population-level prevention and treatment initiatives. A first step in planning for such initiatives is information on the demographic outcomes associated with accomplishing the health objectives of these initiatives. ${ }^{15}$ To this end, the present study models the demographic outlook for Bangladesh from 2015 to 2030 under the assumption of attaining the 2030 SDG target of reducing premature mortality (age $30-70$ years) from four major NCDs by one-third. More specifically, 
we produce the demographic outlook for Bangladesh corresponding to a one-third reduction (ie, $\sim 30 \%$ ) in the unconditional probability of dying between the exact ages of 30 and 70 years from any of CVDs, cancer, diabetes, or chronic respiratory diseases.

\section{METHODS AND DATA}

\section{Patient and public involvement}

Patients or the public were not involved in the design, or conduct, or reporting, or dissemination plans of this research.

\section{The systems-dynamic cohort-component population model}

We develop a cohort-component population projection model that tracks each sex-specific and age-specific cohort of people throughout its lifetime, subject to assumed age-specific and sex-specific mortality, fertility and migration rates. ${ }^{6} 16$ The model represents a 'systems' structure defined by the stocks and flows and the connections between them. ${ }^{17-20}$ In this model, the population in each year is the stock variable, while births, deaths and international migration represent the flows. The model starts with defining the initial-year population, disaggregated by sex and age-cohorts, followed by defining the fertility, mortality and migration attributes of each cohort throughout the projected horizon. In other words, the model in this study resembles an ageing chain where, after birth, each birth cohort progresses from childhood (first stock) to old age (last stock) unless the individual dies and leaves the system.

Figure 1 presents an overview of the model structure using a stock-and-flow diagram. The population's dynamic path begins with the initial population stock observed in 2015 for Bangladesh, disaggregated by sex and age. In each subsequent year, changes in the annual population stock occur through adding births, subtracting deaths and through net international migration (emigration minus immigration) as expressed in Equation 1.

Population dynamics: $P_{s, A+1, t+1}=P_{s, A, t}+B_{s, t}-D_{s, A, t}+N M_{s, A, t}$ (1)

$P_{s, A, t}$ is population by $\operatorname{sex}(s=\{f, m\}), 101$ annual age cohorts $(A=\{0,1,2, \ldots . .100\})$ at year $t=\{0,1,2, \ldots T\}$ (ie, over 2015-2030). $B_{s, t}$ is the number of annual births, determined by applying exogenously set age-specific fertility rates to the cohorts of reproductive-age women (age 15-49).

Births during year: $B_{s, t}=P_{s, A=0, t}=\varnothing_{s} B_{A=0, t}(2)$

Total births: $B_{t}=\sum_{f, A^{*}=15}^{49} B_{A^{*}, t}(3)$

Births by age of mother: $B_{A^{*}, t}=\alpha_{A^{*}, t} P_{f A^{*}, t}(4)$

Where $\varnothing_{s}$ is the probability of $\operatorname{sex}(s=\{f, m\})$ at birth; $\alpha_{A^{*}, t}$ represents age-specific fertility rate for women of reproductive age of $15-49$ years (ie, $A^{*}=\{15,16, \ldots . .49\} \in(f, A)$ ).

In each year, people in each age cohort leave the system due to deaths $\left(D_{s, A, t}\right)$ and net international migration $\left(N M_{s, A, t}\right)$. The causes of deaths are aggregated into six major types of disease categories: CMPN; neoplasms; diabetes; CVDs; respiratory diseases; other NCDs and injuries.
Mortality by sex, age, causes of death: $D_{s, A, d, t}=\delta_{s, A, d, t} P_{s, A, t}(5)$

Where, $\delta_{s, A, d, t}$ represents sex-specific, age-specific and disease-specific death rates over time; $d$ represents the number of deaths from six types of diseases; $d=\{c m p n, n p l, d b t, c v d, r s p, o t h N C D s\}, \quad c m p n: \quad$ communicable, maternal, perinatal, nutritional (WHO Global Health Estimates (GHE) codes I.A., I.B., I.C., I.D., I.E.); $n p l$ : neoplasms (II.A., II.B.); $d b t$ : diabetes (II.C.); cvd: cardiovascular diseases (II.H.); rsp: respiratory diseases (code II.I.); othNCDs: other non-communicable diseases and injuries (II.D., II.E., II.F., II.J., II.K., II.L., II.M., II.N., II.O., II.P., III.A., II.B.). Sex, age-group and diseasespecific deaths rates determine the number of deaths each year. The online supplemental table S1 maps these broad categories with the disaggregated WHO GHE causes of death codes. ${ }^{21}$

Net international migration by sex, age and over time is defined as:

$$
N M_{s, A, t}=P_{s, A, t} * \gamma_{s, A, t}
$$

where $\gamma_{s, A, t}$ is sex and age-specific net international migration rate over the years. Depending on country contexts, sex and age-specific net migration rates determine the number of people removed from (or added to) the population due to migration to (or from) other countries.

The model allows the option of simulating different scenarios by setting sex-specific and age-specific fertility rates; sex-specific, age-specific and disease-specific death rates; and net-migration rates, for each year over the analytical time-horizon (2015-2030). For instance, scenarios of different mortality trends could reflect status-quo (ie, constant death rates over time), trajectories based on historical trends, trajectories based on the predicted impact of disease prevention interventions or reductions in risk factor exposures informed by the literature, or user-defined mortality outlooks based on national plans. We introduce a set of forcing functions $\left(I_{s, A, t}\right)$ with a default or status quo value of 1 but allow scaling-up (scaling-down) functions (over time) corresponding to trend, targets and/or any other implementation sequences:

Death rates scale up/down over time: $\delta_{s, A, d, t} * I_{s, A, d, t}^{\delta}(7)$

Fertility rates scale up/down over time: $\alpha_{A^{*}, t} * I_{s=f, A^{*}, t}^{\alpha}(8)$

Net international migration rate scale up/down over time: $\gamma_{s, A, t} * \tilde{I}_{s, A, t}^{\gamma}(9)$

The model allows user to set scale factors for different years by sex and age-groups. For instance, while $I_{s, d, d, t}^{\delta}=1$ for each year during 2015-2030 in the status quo death rate scenario, $I_{s, A, d, t=2015}^{\delta}=1$ and $I_{s, A, d, t=2030}^{\delta}=0.67$ entail $33 \%$ reduction in the death rates from the 2015 level; scale factors for the interim years (ie, 2016-2029) may include linear interpolated values, or concave/convex path or user-defined values corresponding to interim national targets.

The model generates population counts for 202 annual age-sex cohorts consisting of age $0-100+$ years for men and women, respectively. Data on fertility, mortality and net-migration rates were only available by age-group and 
were assigned to corresponding annual cohorts within each age-group. We used two age-groups depending on data availability, separately for men and women: (1) six broad age-groups: age $0-4$, age $4-14$, age $15-29$, age $30-49$, age $50-69$, age 70 and above; (2) 5 year age-groups: age $0-4$, age $5-9$, age $10-14$, age $15-19$, age $20-24$, age $25-29$, age $30-34$, age $35-39$, age $40-44$, age $45-49$, age $50-54$, age $55-59$, age $60-64$, age 65 and above.

\section{Demographic indicators}

The model produces several key demographic indicators, including population counts and age structure; total, child and old-age dependency ratios; the number of births; crude birth rate; total fertility; net reproduction rate; the rate of natural population increase; the number of deaths by diseases; infant and child mortality rates; crude death rate; life expectancy at birth and at each age; the probabilities of dying between age 30 and 70 ; and total years of lives lost by diseases. The online supplemental table S2 provides brief definitions of the indicators. ${ }^{22}$

\section{BANGLADESH CASE STUDY: DEMOGRAPHIC IMPLICATIONS OF SDG NCD MORTALITY TARGETS \\ Baseline data}

To initiate the population dynamics, we needed base year population, age-specific fertility rates, age-specific death rates and age-specific net migration rates. We used the 2015 annual cohort (age 0-100) population data from the UN population projection (medium variant); age-specific fertility rates reported by the Bangladesh Bureau of Statistics; age-specific total death rates are obtained from the UN life tables for Bangladesh for the year 2015, and agespecific net migration rates from Bangladesh Bureau of Statistics. ${ }^{32-25}$ The UN estimate of the ratio of sex at birth for Bangladesh is 1.05 for their entire analytical horizon; we used the same for this model. ${ }^{25}$ The UN life table for Bangladesh assumes a $100 \%$ mortality rate for ages 85 and above. ${ }^{25}{ }^{26}$ Our model assumed that all people in the last age cohort (100+ years) leave the system (ie, die) with a $100 \%$ mortality rate, with interpolated death rates for ages 85-99. The total net migration rate reported in the UN population projection is $-2.3 / 1000$ population; our model assumed the same statistic when applying age-specific net migration rates to the baseline (2015) population. ${ }^{22}{ }^{25}$ We used WHO GHE disease burden (mortality) data by cause, age and $\operatorname{sex}^{7}$ to decompose the total death rates by six broad categories of diseases, so that, $\delta_{s, A, d, t=2015}=\frac{\text { GHE Death }_{S, A, d, t=2015}}{\sum_{d=1}^{6} \text { GHE Death } s_{S, A, d, t=2015}}$

$$
\begin{gathered}
d=\{c m p n, n p l, d b t, c v d, r s p, o t h N C D s\} \\
\sum_{d=1}^{6} \delta_{s, A, d, t}=\delta_{s, A, t}
\end{gathered}
$$

Where equation 10 is used to decompose the baseline (year=2015) sex-specific and age-specific death rates into six diseases specific rates and GHE Death ${ }_{S, A, d, t=2015}$ represent the number of deaths by diseases obtained from WHO GHE mortality data for the year 2015. $\delta_{s, A, t}$ (ie, sex-specific and age-specific death rates at year $t$ ) is the sum of death rates from six broad categories of diseases $(d)$. The online supplemental table S3 reports the 2015 baseline data used in the model, including the death rates by six broad disease categories.

\section{Scenarios}

We compared three demographic outlooks for Bangladesh: status quo, trend and target. The three scenarios differ in terms of their assumed mortality trajectories, keeping fertility and net migration trajectories the same across scenarios. The UN population projection uses five fertility variants: low, medium, high, constantfertility and instant-replacement-fertility. For instance, for Bangladesh, during 2015-2020 the total fertility rates are assumed to be 2.2, 2.05 and 1.68 for the high, medium and low variants, respectively. For the 20252030 period the total fertility rates are assumed to be 2.26, 1.82 and 1.42 for the high, medium and low variants, respectively. ${ }^{323}$ We use the 2015 age-specific fertility rates reported by the Bangladesh Bureau of Statistics (BBS), setting the total fertility rate at 2.10. Then, using the UN probabilistic projections for age-specific fertility rates for the 2025-2030 period, we scaled down the respective 2015 age-specific fertility rates to arrive at a total fertility rate of 1.82 by 2030 . For the interim years, the model uses interpolated linear trends. We used the 2015 sex-specific and age-specific net migration rates obtained from BBS, which remains constant during the 2015-2030 period. ${ }^{23} 24$

The study uses three variants of mortality trajectories. The 'status quo' scenario entails that the 2015 diseasespecific mortality rates remain constant for the analysis horizon, so that $I_{s, A, t}^{\delta}=1$ for the 2015-2030 period. The 'trend' scenario adopts sex, age-group and diseasespecific mortality rate trajectories based on the latest WHO GHE regional mortality projections for 2016-2030 for Southern Asia, consisting of Bangladesh and other neighbouring countries. ${ }^{27}{ }^{28}$ We estimated the death rates by sex, age-groups and six broad disease categories for 2016 and 2030 from the number of deaths and total population obtained from WHO GHE study; and then produced a matrix of scale factors such that:

$$
I_{s, A, d, t=2030}^{\delta}=\frac{\delta_{S, A, d, t=2030}^{G H E}}{\delta_{S, A, d, t=2016}^{G H E}}
$$

Where $I_{s, A, t=2030}^{\delta}$ are sex-specific, age-specific, and disease-specific scale factors for the death rates in 2030 relative to 2015 levels. The interim years use interpolated scale factors and corresponding mortality rate values. For instance, the WHO GHE estimate projects that by 2030, the death rates of infectious, maternal, perinatal and nutritional conditions would reduce by $21 \%$ for women aged 70 and above and $48 \%$ for men 


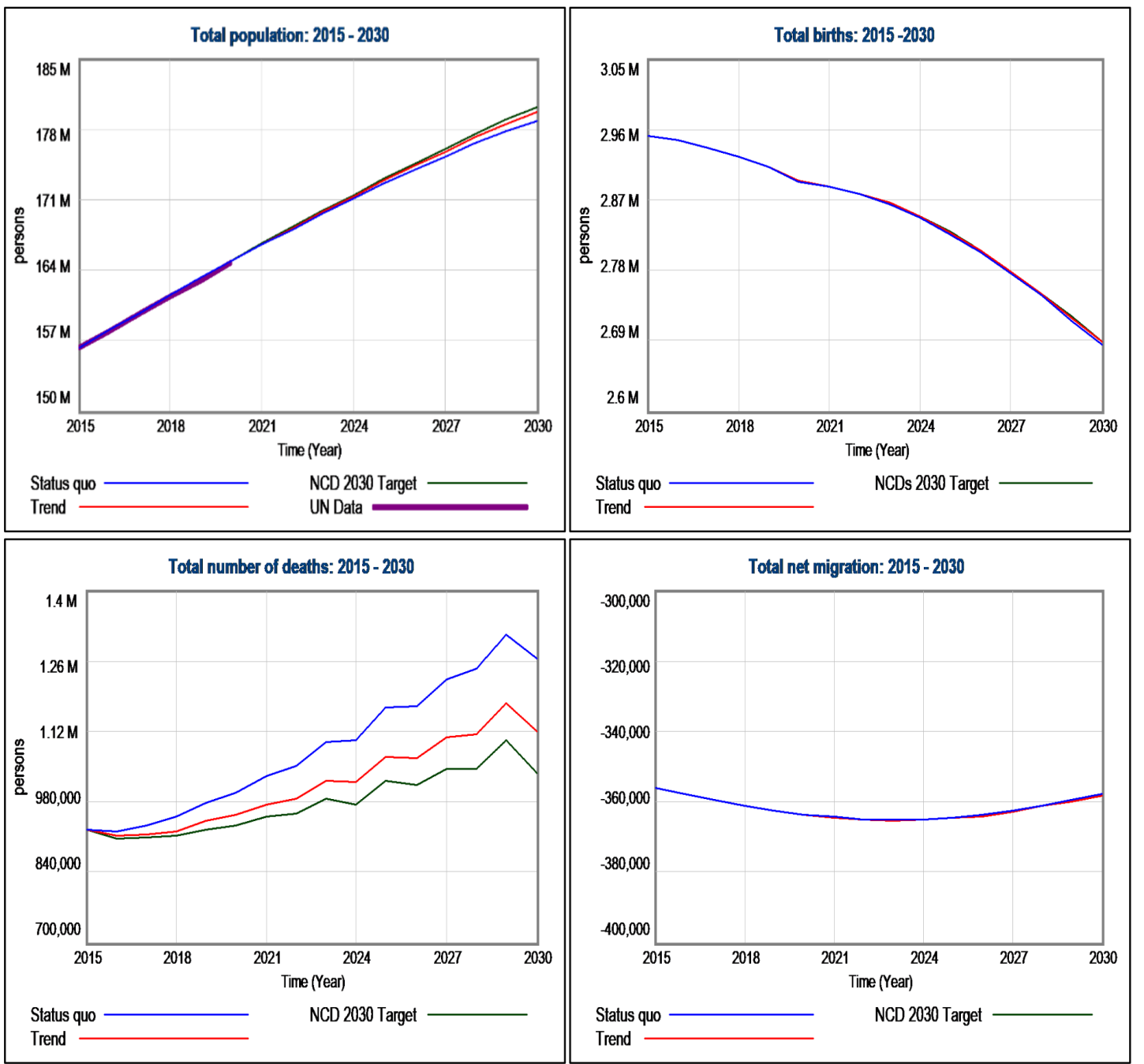

Figure 2 Population outlook for Bangladesh: 2015-2030. Calculations derived from the model. M, million; NCD, noncommunicable disease.

aged 15-29 years. Accordingly, we set the death rate trajectories for the corresponding cohorts to reflect $21 \%$ and $48 \%$ reductions in 2030 from 2015, respectively. Similarly, depending on sex and age-groups, the reductions of death rates range from $3.4 \%$ to $22.9 \%$ for CVDs; $9.3 \%$ to $22.9 \%$ for respiratory diseases; and $3.9 \%$ to $14.1 \%$ for other NCDs and injuries. The trend projections for neoplasms ranges from $9.3 \%$ to $22.9 \%$ increases in the death rates by 2030 . The changes in diabetes death rates ranges from a reduction of $20.7 \%$ to an increase of $3.4 \%$. The online supplemental table $\mathrm{S} 4$ reports all sex, age-group and disease-specific scale factors $\left(I_{s, A, d, t}^{\delta}\right)$ for the trend scenario.

The third scenario is the 'target' scenario, which entails relative reductions in the mortality rates that result in approximately one-third reduction (ie, 30\%) in the unconditional probability of dying between the ages of 30 and 70 years from any one of CVDs, cancer, diabetes or chronic respiratory diseases between 2015 and 2030. For the other two disease categories (ie, CMPN; and other NCDs and injuries) we use the same mortality rate trajectories as in the 'trend' scenario.
The mortality rate trajectory for the four major NCDs follows the trend scenario until 2020 for all age groups, and then declines by 33\% during 2015-2030 for ages 30-69 to arrive at the probability of premature deaths representing about one-third reduction relative to 2015 level; that is, $I_{s, A=30 t 06, d=c c d, r s p, d b, n p h, t=2030}^{\delta}=0.67$ for 2030 and the interim years use interpolated values. The online supplemental table S4 reports the $I_{s, d, d, t}^{\delta}$ values under the target scenarios; the scale factors for 2020 are same in the trend and target scenarios but set at 0.67 for ages 30-69 for 2030 and four NCDs in the target scenario. In achieving the SDG NCD mortality target at the aggregate level, the death rate trajectories can be non-linear and can differ by sex, age and/or disease categories. While the model allows incorporating variants of implementation paths, for the sake of simplicity, in this study we assumed linear interpolated scale factors for the analytical horizon.

This cohort-component systems dynamic population model has been developed using Vensim DSS for Windows V.8.0.4 (Double Precision ×64) (https:// vensim.com/vensim-software/). 


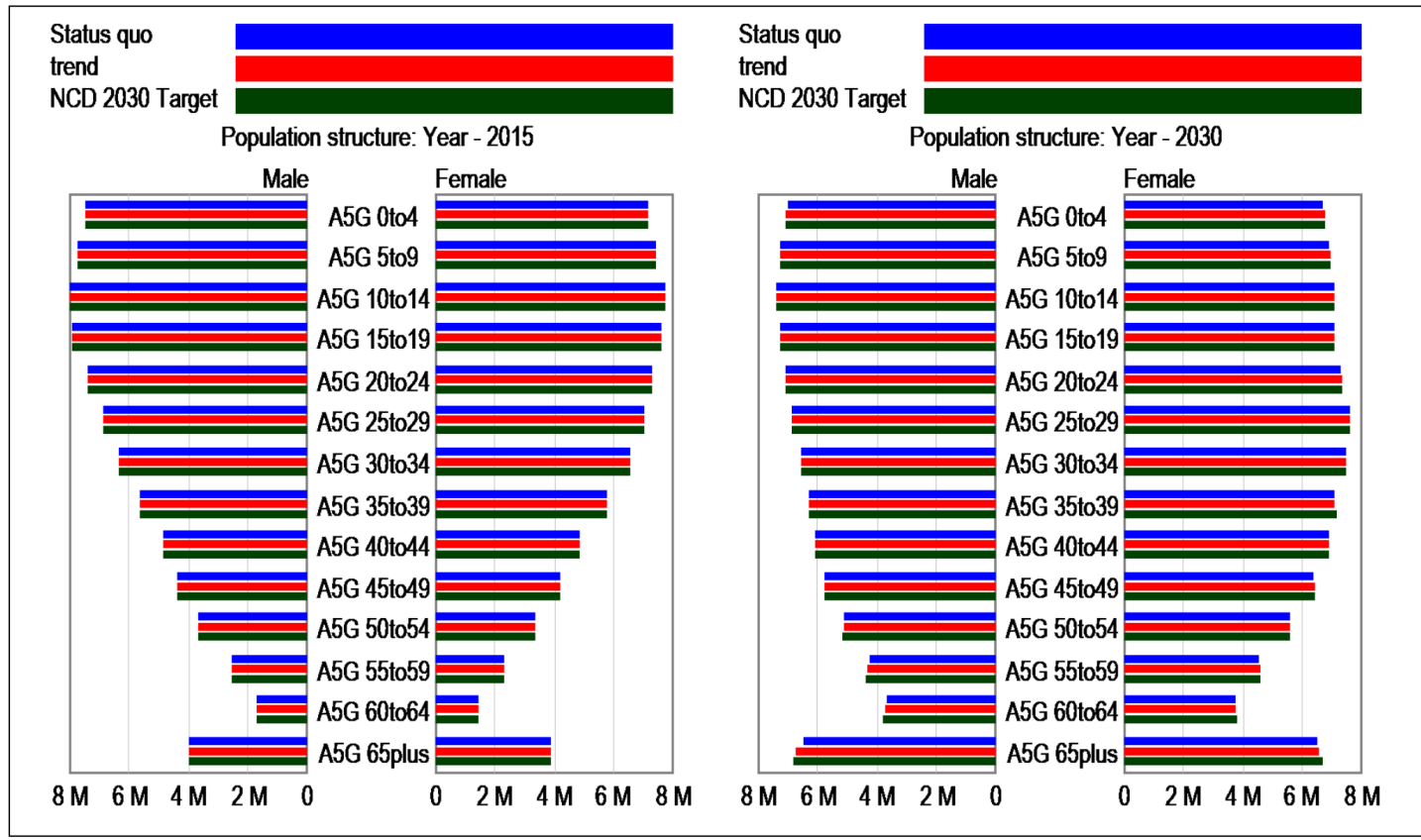

Figure 3 Projected population age structure in Bangladesh: 2015 and 2030. Calculations derived from the model. M, million, NCD, non-communicable disease.

\section{RESULTS}

\section{Population outlooks}

The status quo, trend and target scenarios project 178.9, 179.7 and 180.2 million population in 2030 , respectively. Figure 2 shows the projections for total population along with the three main flow variables in the model, that is, total births, total deaths and total net international migration. Given that all fertility and migration assumptions are the same across all three scenarios, differences in the projected population numbers between scenarios reflect differences in the death rate trajectories. The assumption of a steady decline in total fertility from 2.10 in 2015 to 1.82 in 2030 leads to a declining trajectory for the annual number of births from 2.95 million in 2015 to around 2.69 million in 2030. The assumed constant age-specific net migration rate kept the total number of people migrating abroad between 356000 and 365000 each year. However, the annual number of deaths is much higher in the status quo scenario compared with the trend and target scenarios. The model projects 1.26, 1.12 and 1.04 million deaths in 2030 in the status quo, trend and target scenario, respectively. The cumulative number of deaths during 2015-2030 are 17.37, 16.22 and 15.64 million in the status quo, trend and the target scenario, entailing 1.73 million and 584000 deaths averted in the target scenario compared with the status quo and trend scenarios, respectively.

Figure 3 shows the inverted age-sex pyramid illustrating the distribution of various age groups in Bangladesh in 2015 (left panel) and 2030 (right panel). The population is distributed along the horizontal axis, with men shown on the left and women on the right. The male and female populations are broken down into 5-year age groups represented as horizontal bars along the vertical axis, with the youngest age groups (age 0-4) at the top and the oldest at the bottom (age 65 and above). The shape of the population pyramid gradually evolves during 2015-2030 based on fertility, mortality and international migration

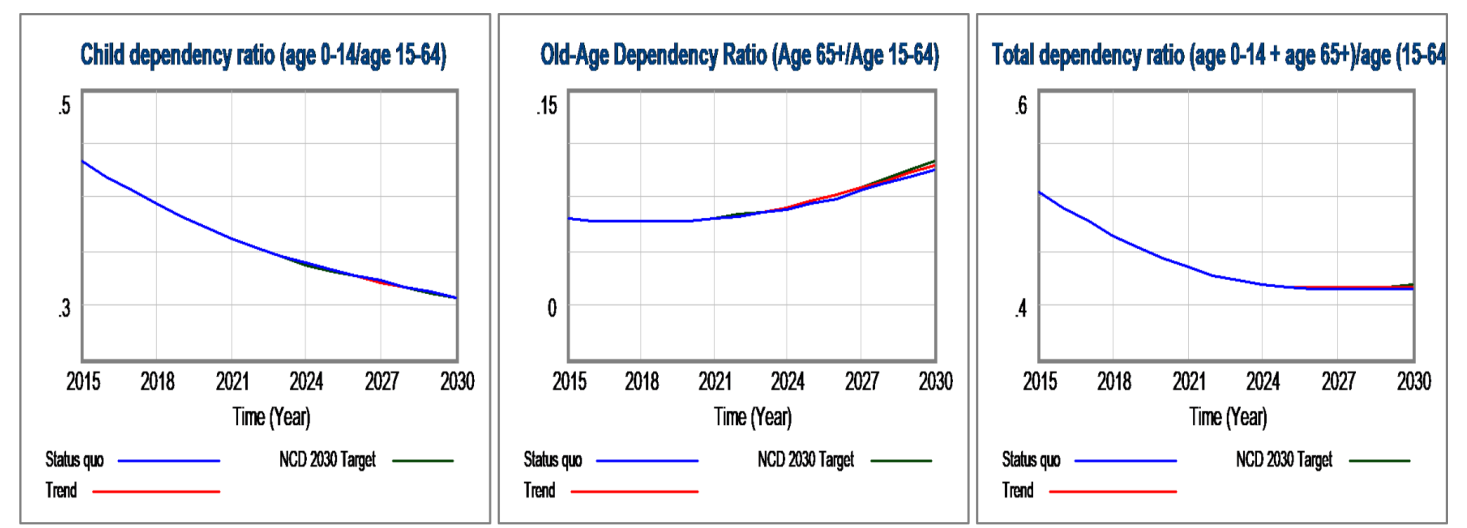

Figure 4 Child, old-age and total dependency ratios. Calculations derived from the model. NCD, non-communicable disease. 


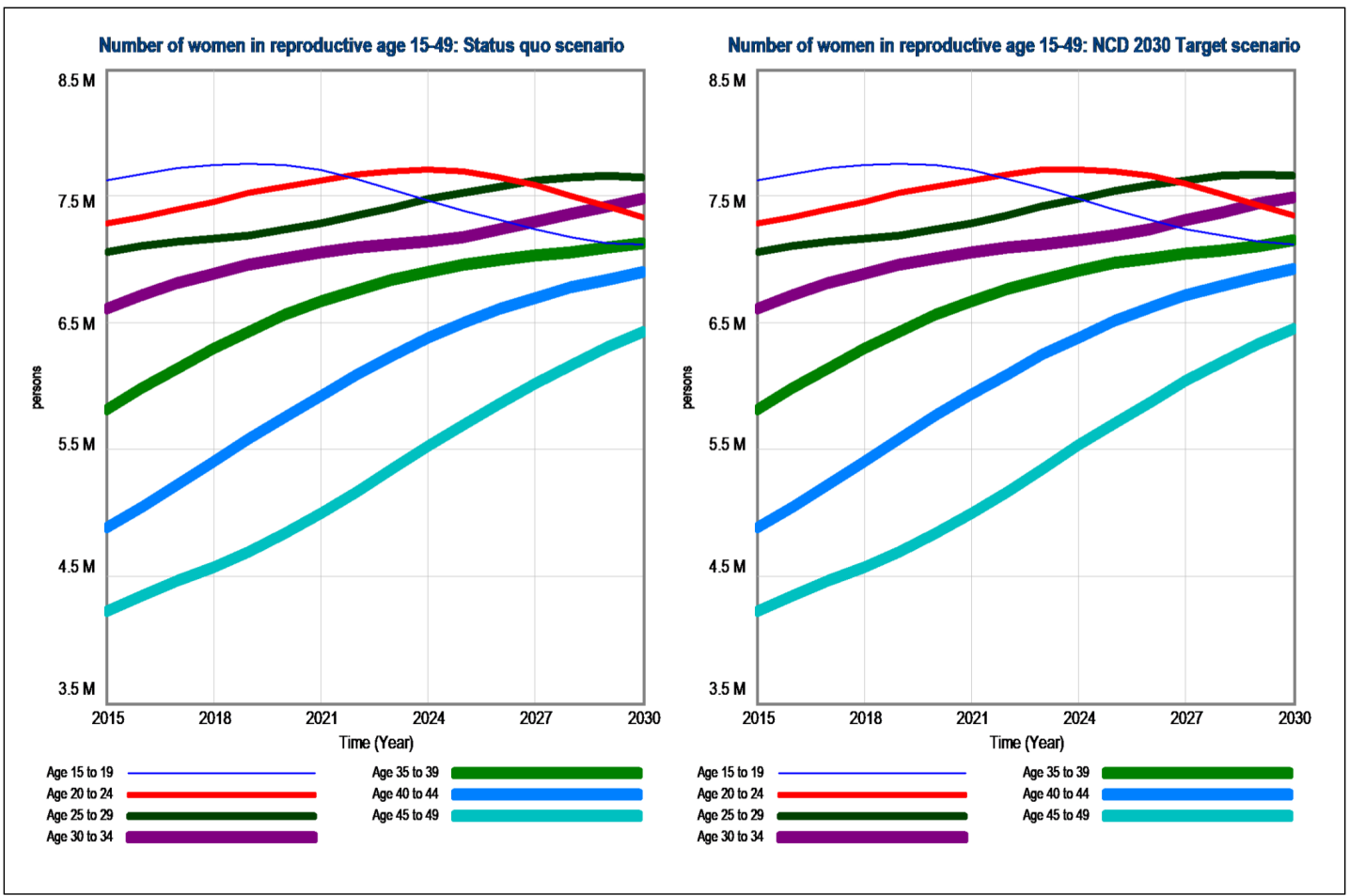

Figure 5 Number of women in reproductive age (15-49 years). Calculations derived from the model. M, million; NCD, noncommunicable disease.

trends. The apparent cone-shaped population pyramid in 2015 appears more symmetric in 2030, consistent with population ageing over the analytical horizon.

The evolving population structure is also reflected in figure 4. The rapid reductions in infant and child mortality accompanied by decreasing fertility led to a continuous reduction in the child dependency ratio (ie, ratio of population age $0-14$ and age 15-64) (0.45 in 2015 vs 0.35 in 2030 trend scenario). On the other hand, as the annual cohorts progress through the analytical period, the old-age dependency ratio (ie, ratio of population age 65 and above and age 15-64), after remaining relatively flat during 2015-2020, starts to rise beyond 2020 (0.078 in 2015; 0.077 in 2020; and $~ 0.10$ in 2030 for the three scenarios). The total dependency ratio (ie, ratio of population age 0-14 and age 65 and above, and age 15-64) registers a relatively quick decline from 0.52 in 2015 to 0.45 in 2025 and remains at 0.45 until 2030.

The annual number of births is determined by the agespecific fertility rates and the number of women of reproductive age 15-49 years. The trajectory of the number of women in reproductive age is affected by the number of deaths and international migration for the corresponding cohorts. In figure 5 , for the trend and target scenarios, it is evident that the number of women aged 15-19 begins to decline after 2021, and the number of women aged 20-24 declines after 2024. The number of women in all other older age groups increases during 2015-2030, with older cohorts showing larger growth.

Figure 6 presents the projected mortality trajectories by disease categories. The number of deaths from all disease categories increases except for the CMPN category in the status quo scenario. In the status quo scenario, population decreases moderately for younger cohorts (ie, age <25) and increases more for the older cohorts age 25 and above during 2015-2030 period, leading to net increase in the total population. Consequently, the assumed constant death rates for the CMPN in the status quo scenario results in net increase in total deaths from CMPN. On the other hand, the continuous decline in death rates and a near-flat population trend with a slight decrease in numbers of children and adolescents lead to a reduction in deaths from CMPN in the trend and target scenarios. In all scenarios, NCD deaths rise with the rising population; however, the number of deaths is much smaller in the target scenario. The share of CMPN in total deaths declines from $26 \%$ in 2015 to $23 \%, 17.6 \%$, and $19.1 \%$ in 2030 under the status quo, trend and target scenario, respectively. On the other hand, the contribution of the four major NCDs (CVD, respiratory diseases, diabetes and neoplasms) in total deaths increases from $54.9 \%$ in 2015 to $58.9 \%, 63.4 \%$ and $60.2 \%$ in 2030 under the status quo, trend and target scenarios, respectively.

Table 1 shows the number of deaths under the three mortality scenarios and the number of deaths averted under the target scenario compared with the status quo and trend. Of the four major NCDs, CVD is the major killer, followed by neoplasm, respiratory diseases and diabetes. In 2025, the model projects 375000,357000 and 334000 deaths from CVD under status quo, trend and target, respectively, which entails 23000 and 41000 CVD deaths averted under the target scenario compared 


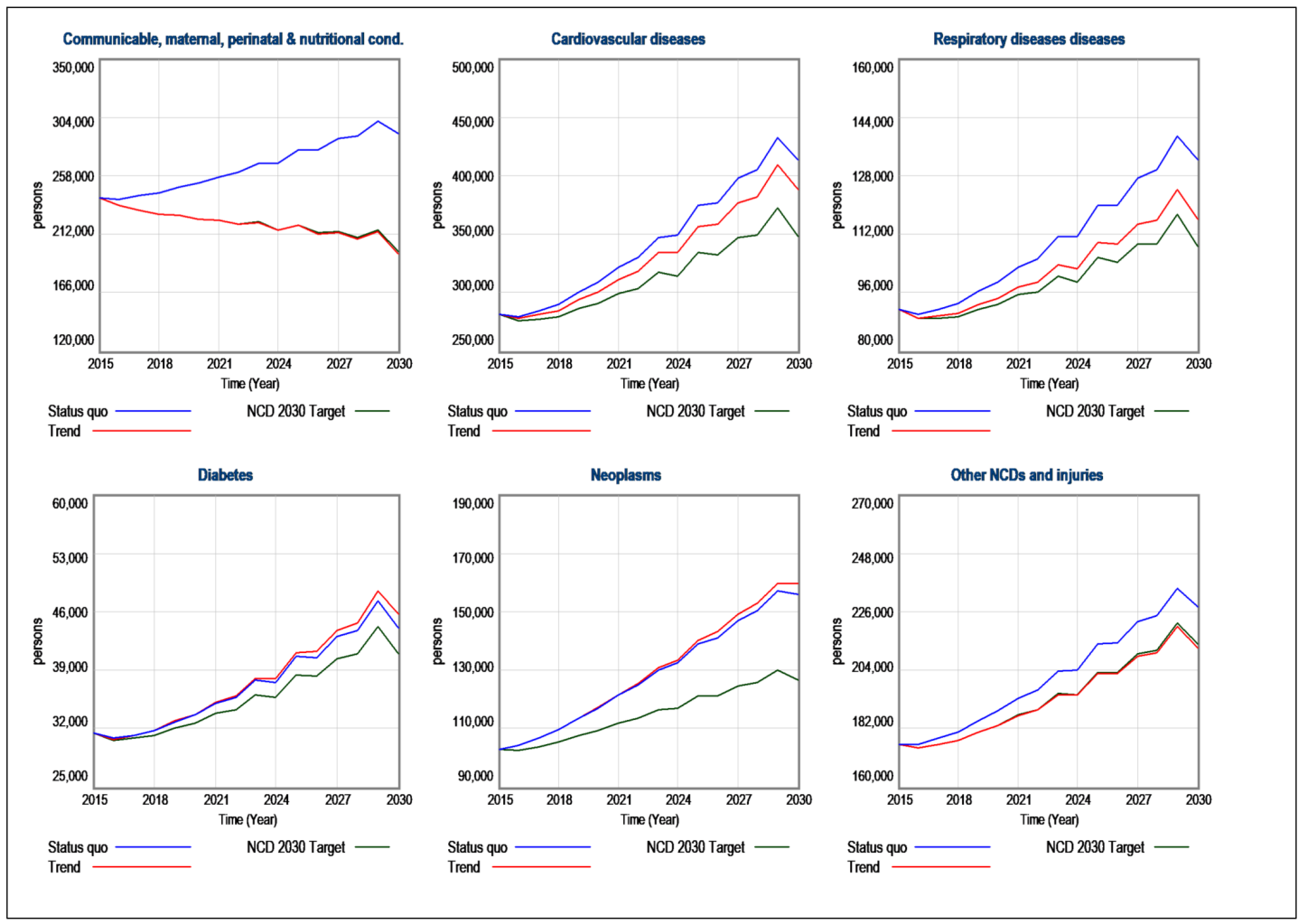

Figure 6 Mortality by diseases. Calculations derived from the model. NCD, non-communicable disease.

with trend and status quo scenarios. Over 2015-2030, the target scenario would avert a cumulative 485000 (285000 men and 199000 women) CVD deaths and 282000 CVD deaths (162000 men and 120000 women) compared with the status quo and trend scenario, respectively. Under the target scenario, the cumulative (2015-2030) number of deaths averted from the four major NCDs is projected to be about 897000 (500 000 men and 396000 women) and 596000 (291000 men and 305000 women), compared with the status quo and trend scenarios, respectively.

The online supplemental table S5 shows the projections of years of lives lost (YLL) in the three scenarios, and YLL averted in the target scenario compared with status quo and trend. Compared with the status quo mortality trajectories, the attainment of NCD targets would avert a cumulative (2015-2030) 14.9 million YLL (ie, 7.74, 2.2, 0.49 and 4.49 million YLL averted form CVD, respiratory diseases, diabetes and neoplasm, respectively). Compared with the trend mortality trajectories, the attainment of NCD targets would avert a cumulative (2015-2030) 12.16 million YLL (ie, 5.30, 0.92, 0.64 and 5.30 million YLL averted form CVD, respiratory diseases, diabetes and neoplasm, respectively).

Table 2 reports the projections for life expectancy, infant mortality, under-five mortality and probability of premature deaths (ie, between age 30-70) from NCDs. Male life expectancy at birth increases from 71.10 in 2015 to 73.47 and 74.38 years in 2030 under the trend and target scenario, respectively. Female life expectancy at birth increases from 73.68 years in 2015 to 75.34 and 76.39 in 2030 in the trend and target scenarios. Fulfilment of SDG NCD mortality targets entail 2.63 and 1.96 year increases in life expectancy at age 30 for male and female population, respectively (ie, life expectancies at 30 in the target scenario: 43.79 years in 2015 vs 46.42 years in 2030 for men; and 46.36 years in 2015 vs 48.32 years in 2030 for women). The projections show declining trends for infant and child mortality in both trend and target scenarios. Since the drivers of infant and child mortality are primarily CMPN diseases, the magnitudes of reduction are similar in the trend and target scenarios. Large reductions in the probabilities of premature deaths (ie, between age 30-70) are projected in both scenarios, and the reduction is much larger in the target scenario. The probability of death for men between age 30-70 from any of four major NCDs decreases from 219 per 1000 people in 2015 to 198 and 153 per 1000 people in 2030 in the trend and target scenarios, respectively. The probability of premature death for women from four major NCDs decreases from 199 per 1000 people in 2015 to 186 and 138 per 1000 people in 2030 in the trend and target scenarios, respectively. For the target scenario, these entail an overall $30 \%$ reduction in the probability of premature deaths from four major NCDs.

\section{DISCUSSION}

The cohort-component model in this study projects the demographic outlook of a population using a systemsdynamic process determined by inter-relationships between population determinants, including those affected by policy actions. ${ }^{21729}$ The strengths of this 
Table 1 Number of deaths and deaths averted from four major NCDs

\begin{tabular}{|c|c|c|c|c|c|c|c|c|c|}
\hline & \multicolumn{3}{|c|}{$\begin{array}{l}\text { Deaths and deaths averted in } \\
\qquad 2025\end{array}$} & \multicolumn{3}{|c|}{$\begin{array}{l}\text { Deaths and deaths averted in } \\
\qquad 2030\end{array}$} & \multicolumn{3}{|c|}{$\begin{array}{l}\text { Cumulative number of deaths and } \\
\text { deaths averted: } 2015-2030\end{array}$} \\
\hline & Male & Female & Total & Male & Female & Total & Male & Female & Total \\
\hline \multicolumn{10}{|c|}{ Cardiovascular diseases } \\
\hline \multicolumn{10}{|l|}{ Number of deaths } \\
\hline Status quo & 189239 & 185322 & 374561 & 212078 & 201626 & 413704 & 2790691 & 2701365 & 5492056 \\
\hline Trend & 178532 & 178423 & 356955 & 197107 & 191310 & 388417 & 2667253 & 2621899 & 5289152 \\
\hline \multicolumn{10}{|c|}{ Deaths averted in NCD target scenario } \\
\hline Compared with status quo & 24121 & 16753 & 40874 & 38039 & 28205 & 66244 & 285214 & 199336 & 484550 \\
\hline Compared with trend & 13414 & 9854 & 23268 & 23068 & 17889 & 40957 & 161776 & 119870 & 281646 \\
\hline \multicolumn{10}{|c|}{ Respiratory diseases } \\
\hline NCD target & 58953 & 46487 & 105439 & 61818 & 46678 & 108496 & 894714 & 692819 & 1587533 \\
\hline \multicolumn{10}{|c|}{ Deaths averted in NCD target scenario } \\
\hline Compared with status quo & 8809 & 5732 & 14540 & 13873 & 9931 & 23804 & 104084 & 68670 & 172755 \\
\hline Compared with trend & 1633 & 2717 & 4350 & 2727 & 4962 & 7689 & 19545 & 33082 & 52627 \\
\hline \multicolumn{10}{|c|}{ Diabetes } \\
\hline \multicolumn{10}{|l|}{ Number of deaths } \\
\hline Status quo & 16036 & 24649 & 40685 & 17809 & 26248 & 44056 & 236911 & 360091 & 597002 \\
\hline Trend & 15686 & 25412 & 41097 & 17613 & 28072 & 45685 & 233429 & 370221 & 603650 \\
\hline NCD target & 14858 & 23602 & 38460 & 16199 & 24798 & 40997 & 223476 & 348223 & 571698 \\
\hline \multicolumn{10}{|c|}{ Deaths averted in NCD target scenario } \\
\hline \multicolumn{10}{|c|}{ Neoplasm } \\
\hline Trend & 75386 & 64846 & 140232 & 85322 & 74376 & 159698 & 1119111 & 951193 & 2070304 \\
\hline NCD target & 67086 & 54086 & 121172 & 71225 & 55565 & 126789 & 1019066 & 820728 & 1839794 \\
\hline \multicolumn{10}{|c|}{ Deaths averted in NCD target scenario } \\
\hline Compared with status quo & 8281 & 9676 & 17957 & 12735 & 16441 & 29176 & 97407 & 116551 & 213958 \\
\hline Compared with trend & 8300 & 10759 & 19060 & 14098 & 18811 & 32909 & 100045 & 130465 & 230510 \\
\hline
\end{tabular}

Calculations derived from the model.

$\mathrm{NCD}$, non-communicable disease.

model are several. First, it is replicable as it uses established principles about the dynamics of the population process. Second, it can produce consistent and comparable cross-country estimates that are easy to update using country data across multiple countries. Third, it can provide focused estimates for target groups of interest because it tracks population outcomes at a highly disaggregated level. In the same vein, the model can be flexibly adapted to the intended disaggregation schemes (eg, more aggregate) of population cohorts and disease categories; Finally, the model outcomes can be potentially linked to other dynamic inputs related to health systems, education, the environment, housing and city planning, infrastructure, energy and utility and alike. ${ }^{29}$ The main contribution of the model used in this study is in estimating the expected demographic shifts associated with different disease-specific mortality trajectories. The resulting estimates inform the effects of proposed NCD control targets, linking the number of deaths averted by achieving these targets to demographic shifts in the population. $^{29}$

The model in this study has several limitations. The cohort-component method does not explicitly incorporate socio-economic determinants of population change. The evolution of fertility, mortality and migration over time are not endogenously determined; the respective 
Table 2 Life expectancy, infant mortality rate, under-five mortality, probability of dying age 0-70

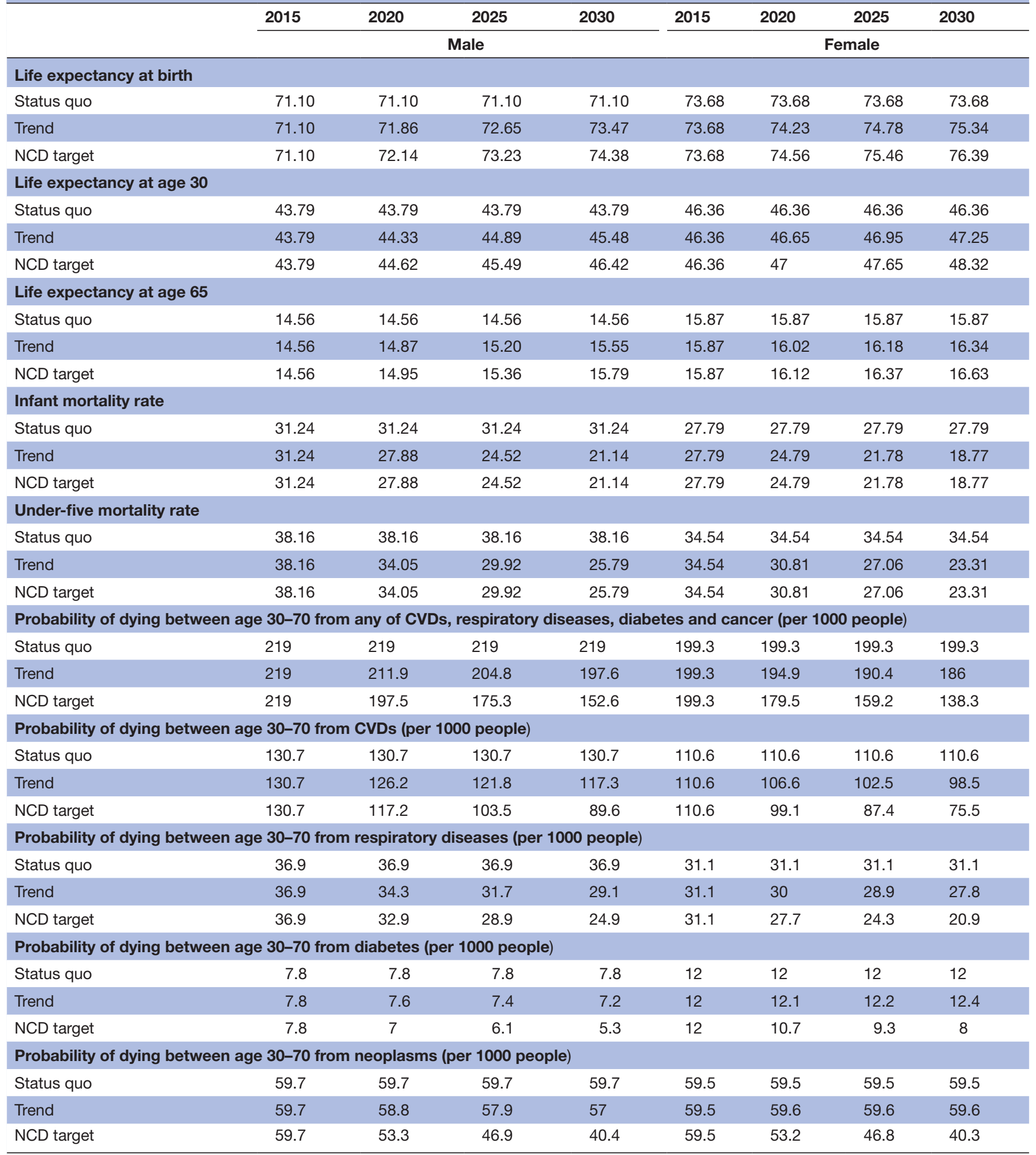

Calculations derived from the model.

CVD, cardiovascular disease; NCD, non-communicable disease.

trajectories are set exogenously using informed assumptions. To that effect, the model outcomes are projections based on a set of assumptions about trajectories of mortality, fertility and migration. The objective is not to make a perfect prediction of the future, but to assess comparative differences in population trajectories resulting from different health policy scenarios, keeping other input assumptions constant. Therefore, the model 
outcomes should not be interpreted as a perfect forecast but are based on conditional calculations showing what the future population would be if a particular set of reasonable assumptions were to hold true. Using similar assumptions but different approach, a global study by Cao et al quantified the potential gains in average expected life-years lived between 30 years and 70 years of age worldwide should the SDG target of a one-third reduction in premature mortality from the four major NCDs be achieved, as well as the maximum gains if all premature mortalities from these diseases were eliminated. ${ }^{30}$ While the model in our paper captures differences in mortality scenarios, it does not capture the extent of disabilities averted from attaining the targets. Also, the scenarios do not consider the mortality implications of the recent COVID-19 pandemic in Bangladesh.

The model generates the evolution of annual cohorts and population structure during 2015-2030 using demographic indicators for Bangladesh that are consistent with those offered by international agencies. ${ }^{3-5}$ For instance, while the model replicates the baseline (2015) demographic indicators as reported in UN population projections, the population shares in 2030 for the $0-14,15-64$ and 65 and above years old age-groups in the UN medium variant projections versus the model trend projections compare as follow: 22.9 versus 23.8; 69.7 versus 68.8 ; and 7.4 versus 7.4, respectively. This model captures dynamic population outflows based on deaths from disaggregated disease categories, allowing comparison between diseasespecific mortality scenarios. We estimated that by attaining NCD targets in compliance with SDG 2030 goals, people in Bangladesh will live longer by more than 3 years on average (3.28 and 2.71 years for men and women, respectively). Over the 15-year analysis period, a cumulative 1.73 million all-cause deaths (99 600 men and 73600 women) and 584000 all-cause deaths (284 000 men and 300000 women) would be averted in the NCD target scenario compared with the status quo and trend scenarios, respectively. In the target scenario, the cumulative number of deaths averted from the four major NCDs are projected to be 896000 (500 000 men and 396000 women) and 597 000 (291000 men and 305000 women), compared with the status quo and trend scenarios, respectively. These estimates inform the potential benefits as well as tradeoffs in health and demographic outcomes associated with accomplishing current NCD targets.

Contributors MJH conceptualised the study, implemented the methodology, developed the modelling framework in the Vensim software, acquired data and led the formal analysis and write-up. BKD contributed to the study plan and analysis, model development, interpretation of results and critical review of the paper. DK contributed to the study plan and analysis, interpretation of results and critical review of the paper. All authors critically reviewed the manuscript and approved the final version.

Funding The authors have not declared a specific grant for this research from any funding agency in the public, commercial or not-for-profit sectors.

Disclaimer The findings and conclusions of this report are those of authors only and do not necessarily represent the official position of the Centers for Disease Control and Prevention.
Competing interests None declared.

Patient consent for publication Not required.

Provenance and peer review Not commissioned; externally peer reviewed.

Data availability statement Data are reported in the supplemental file; and also publicly available in open access repository and/or published reports (see references cited).

Supplemental material This content has been supplied by the author(s). It has not been vetted by BMJ Publishing Group Limited (BMJ) and may not have been peer-reviewed. Any opinions or recommendations discussed are solely those of the author(s) and are not endorsed by BMJ. BMJ disclaims all liability and responsibility arising from any reliance placed on the content. Where the content includes any translated material, BMJ does not warrant the accuracy and reliability of the translations (including but not limited to local regulations, clinical guidelines, terminology, drug names and drug dosages), and is not responsible for any error and/or omissions arising from translation and adaptation or otherwise.

Open access This is an open access article distributed in accordance with the Creative Commons Attribution Non Commercial (CC BY-NC 4.0) license, which permits others to distribute, remix, adapt, build upon this work non-commercially, and license their derivative works on different terms, provided the original work is properly cited, appropriate credit is given, any changes made indicated, and the use is non-commercial. See: http://creativecommons.org/licenses/by-nc/4.0/.

ORCID iD

Muhammad Jami Husain http://orcid.org/0000-0001-6049-0505

\section{REFERENCES}

1 Shryock HS, Siegel JS, Larmon EA. The methods and materials of demography. 2. US Bureau of the Census, 1973.

2 Siegel J, Swanson D, eds. The Methods and Materials of Demography. 2nd edn. San Diego: Elsevier Academic Press, 2004.

3 United Nations, Department of Economic and Social Affairs, Population Division. World population prospects 2019: methodology of the United nations population estimates and projections, 2019. Available: https://population.un.org/wpp/Publications/Files/ WPP2019_Methodology.pdf [Accessed 5 Apr 2020].

4 The World Bank. Population estimates and projections. data catalog. Available: https://datacatalog.worldbank.org/dataset/populationestimates-and-projections [Accessed 5 Apr 2020].

5 The U.S. Census Bureau. International data base: population estimates and projections methodology, 2013. Available: https:// www2.census.gov/programs-surveys/international-programs/ technical-documentation/methodology/idb-methodology.pdf [Accessed 5 Apr 2020].

6 O'Neill BC, Balk D, Brickman M, et al. A guide to global population projections. Demogr Res 2001;4:203-88.

7 World Health Organization.. Global health estimates 2016: disease burden by cause, age, sex, by country and by region, 2000-2016. Geneva: World Health Organization, 2018.

8 United Nations. Transforming our world: The 2030 agenda for sustainable development. General Assembley 70 session 2015.

9 World Health Organization. Global status report on noncommunicable diseases 2014 (No. WHO/NMH/NVI/15.1). World Health Organization, 2014.

10 World Health Organization. 13th General programme of work (GPW13) WHO impact framework. Geneva: World Health Organization, 2018. https://www.who.int/about/what-we-do/GPW13 WHO_Impact_Framework_Indicator_Metadata.pdf

11 El-Saharty S, Ahsan KZ, Koehlmoos TL, et al. Tackling noncommunicable diseases in Bangladesh: now is the time. The World Bank, 2013.

12 Biswas T, Pervin S, Tanim MIA, et al. Bangladesh policy on prevention and control of non-communicable diseases: a policy analysis. BMC Public Health 2017;17:582.

13 Government of People's Republic of Bangladesh. Multisectoral action plan for prevention and control of noncommunicable diseases 2018-2025 with a three-year operational plan. May 2018. Noncommunicable Disease Control Programme, Directorate General of Health Services, Health Services Division, Ministry of Health \& Family Welfare. Available: https://www.who.int/docs/default-source/ searo/ncd/ban-ncd-action-plan-2018-2025.pdf?sfvrsn=f281d939_2

14 World Health Organization. Global action plan for the prevention and control of noncommunicable diseases 2013-2020. WHO, 2013. Available: http://apps.who.int/iris/bitstream/10665/94384/1/ 9789241506236_eng.pdf 
15 World Health Organization. Tackling NCDs: 'best buys' and other recommended interventions for the prevention and control of noncommunicable diseases (No. WHO/NMH/NVI/17.9). World Health Organization, 2017.

16 George MVT, Smith SK, Swanson DA. Population projections. Chapter 21. In: Siegel J, Swanson D, eds. The methods and materials of demography. San Diego: Elsevier Academic Press, 2004.

17 García JM. Theory and practical exercises of system dynamics: modeling and simulation with Vensim PLE. Preface John Sterman. Juan Martin Garcia, 2020.

18 Homer JB, Hirsch GB. System dynamics modeling for public health: background and opportunities. Am J Public Health 2006;96:452-8.

19 Atkinson J-A, Page A, Prodan A, et al. Systems modelling tools to support policy and planning. Lancet 2018;391:1158-9.

20 Luke DA, Stamatakis KA. Systems science methods in public health: dynamics, networks, and agents. Annu Rev Public Health 2012;33:357-76.

21 World Health Organization. WHO methods and data sources for global burden of disease estimates 2000-2016. Global health estimates technical paper WHO/HIS/IER/GHE/2018.4. Geneva: WHO, 2018.

22 United Nations, Department of Economic and Social Affairs, Population Division. World population prospects: the 2012 revision, 2013.

23 Bangladesh Bureau of Statistics. Population Projection of Bangladesh: Dynamics and Trends 2011-2016. Statistics and Informatics Division (SID), Ministry of Planning, Government of the People's Republic of
Bangladesh, 2015. http://203.112.218.65:8008/WebTestApplication/ userfiles/Image/PopMonographs/PopulationProjection.pdf

24 Bangladesh Bureau of Statistics. Statistical year book Bangladesh 2018. Dhaka, Bangladesh: Statistics and Information Division (SID), Ministry pf Planning, Government of the People's Republic of Bangladesh, 2019.

25 World Health Organization. Life tables. Global health Observatory (GHO) data. Life expectancy, 2020. Available: https://www.who.int/ gho/mortality_burden_disease/life_tables/life_tables/en/ [Accessed 6 Apr 2020].

26 World Health Organization. WHO methods and data sources for life tables 1990-2015. Department of Information, Evidence and research. Global health estimates technical paper WHO/HIS/IER/ GHE/2016.2. WHO, Geneva.

27 World Health Organization. Projections of mortality and causes of death, 2016 to 2060 . Health statistics and information systems. Global health estimates. Available: https://www.who.int/healthinfo/ global burden disease/projections/en/ [Accessed 6 Apr 2020].

28 Mathers CD, Loncar D. Projections of global mortality and burden of disease from 2002 to 2030. PLoS Med 2006;3:e442.

29 Currie DJ, Smith C, Jagals P. The application of system dynamics modelling to environmental health decision-making and policy - a scoping review. BMC Public Health 2018;18:402.

30 Cao B, Bray F, Ilbawi A, et al. Effect on longevity of one-third reduction in premature mortality from non-communicable diseases by 2030: a global analysis of the sustainable development goal health target. Lancet Glob Health 2018;6:e1288-96. 